01

\title{
Спин-обменные столкновения в системе K-Li
}

\author{
(C) В.А. Картошкин \\ ФТИ им. А.Ф. Иофрфе РАН, \\ 194021 Санкт-Петербург, Россия \\ e-mail:victor.kart@mail.ioffe.ru
}

Поступила в редакцию 15.03.2019 г.

В окончательной редакции 15.03.2019 г.

Принята к публикации 20.06.2019 г.

\begin{abstract}
Впервые проведен расчет сечений спинового обмена и сдвига частоты магнитного резонанса при столкновении атомов лития и калия в основном состоянии. Расчет сечений проведен на основании данных по синглетному $\left(X^{1} \Sigma^{+}\right)$и триплетному $\left(a^{3} \Sigma^{+}\right)$потенциалам взаимодействия димера ${ }^{39} \mathrm{~K}^{7} \mathrm{Li}$. Переход от энергетических к температурным зависимостям действительной и мнимой частей комплексного сечения спинового обмена позволяет получить информацию как об уширении линии магнитного резонанса исследуемых атомов, так и о сдвиге частоты магнитного резонанса при их столкновении.
\end{abstract}

Ключевые слова: спиновый обмен, поперечные сечения.

DOI: $10.21883 /$ OS.2019.10.48369.107-19

\section{Введение}

Интерес к исследованию спин-обменных процессов при столкновении атомов щелочных металлов обусловлен, в частности, тем, что щелочные атомы (Cs [1], $\mathrm{Rb}$ [2], K [3]) могут быть использованы в качестве рабочей среды в квантовых магнитометрах с оптической накачкой [4]. При этом могут быть использованы атомы как одного сорта, например Cs [1], так и смесь, например $\mathrm{K}-\mathrm{Cs}$ [5]. Точностные характеристики подобного рода приборов определяются, в частности, ширинами линий магнитного резонанса оптически ориентированных атомов, а также сдвигами частот магнитного резонанса, обусловленными спин-обменными столкновениями. Для определения влияния спин-обменных стокновений на ширины линий магнитного резонанса и на сдвиги линий магнитного резонанса необходимо знание комплексных сечений спинового обмена [6]. При этом действительная часть комплексного сечения определяет передачу поляризации в столкновении, релаксацию [7] и образование высших поляризационных моментов [8]. Мнимая часть сечения определяет сдвиг частоты магнитного резонанса в системе зеемановских и сверхтонких уровней атомов [9].

Для оценки вкладов спинового обмена в ширину и сдвиг частоты сигналов магнитного резонанса необходимо знание температурных зависимостей комплексного сечения спинового обмена в системе сталкивающихся атомов. В настоящей работе впервые проведен расчет этих сечений на основании потенциалов взаимодействия димера ${ }^{39} \mathrm{~K}^{7} \mathrm{Li}[10]$.

\section{Синглетный и триплетный потенциалы взаимодействия молекулы KLi}

Синглетный и триплетный термы, описывающие взаимодействие атомов $\mathrm{K}$ и $\mathrm{Li}$ в основном состоянии, были представлены в [10]. В этой работе методом фурье-спектроскопии высокого разрешения были получены данные, позволившие построить синглетный $X^{1} \Sigma^{+}$и триплетный $a^{3} \Sigma^{+}$потенциалы взаимодействия димера KLi.

Были определены энергия диссоциации молекулы $\mathrm{KLi}$ для синглетного терма $D_{e}=6216.886 \pm 1.5 \mathrm{~cm}^{-1}$ и равновесное расстояние $\mathrm{R}_{e}=3.1914 \AA$, для триплетного терма $T_{e}=287.0 \pm 1.5 \mathrm{~cm}^{-1}$ и равновесное расстояние $\mathrm{R}_{e}=4.9915 \AA$ (в атомной системе единиц эти величины имеют следующие значения: $D_{e}=0.0283$ a.u., $\mathrm{R}_{e}=6.023 a_{0} ; T_{e}=0.00131$ a.u., $\left.\mathrm{R}_{e}=9.421 a_{0}\right)$.

Как отмечалось выше, образующаяся в процессе столкновения двухатомная молекула описывается в основном состоянии синглетным термом с полным моментом $S=0$ и триплетным термом с полным моментом $S=1$. В [10] синглетный и триплетный потенциалы взаимодействия были представлены в аналитическом виде для трех областей межъядерных расстояний.

Для малых межъядерных расстояний $\left(R<R_{S R}\right)$ потенциал имел вид отталкивания:

$$
U_{S R}(R)=A+\frac{B}{R^{q}} .
$$

Для больших межьядерных расстояний $\left(R>R_{L R}\right)$ потенциал описывался ван-дер-ваальсовым взаимодействием с учетом члена, описывающего обменное взаимодействие:

$$
U_{L R}=-\frac{C_{6}}{R^{6}}-\frac{C_{8}}{R^{8}}-\frac{C_{10}}{R^{10}} \pm E_{e x} .
$$

Здесь $E_{e x}$ - обменное взаимодействие, явный вид которого представлен в [10]. Обменное взаимодействие входит со знаком $(+)$ в триплетный и со знаком (-) в синглетный термы:

$$
E_{e x}=A_{e x} R^{\gamma} \exp (-\beta R),
$$




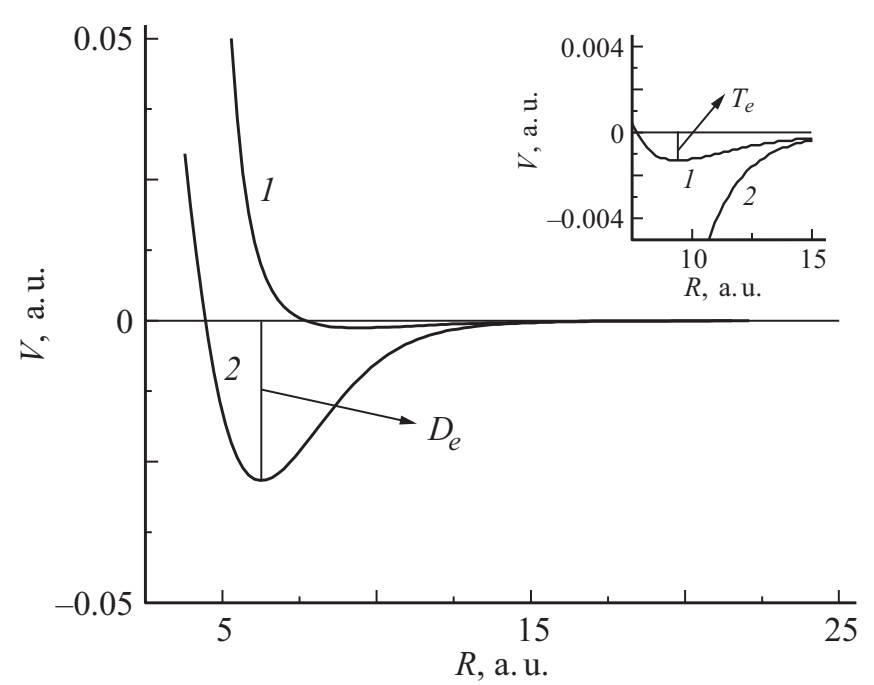

Рис. 1. Потенциалы взаимодействия атомов ${ }^{39} \mathrm{~K}-{ }^{7} \mathrm{Li}: 1-$ синглетный $\left(X^{1} \Sigma^{+}\right), 2-$ триплетный $\left(a^{3} \Sigma^{+}\right)$термы по данным [10].

Для области $\left(R_{S R}<R<R_{L R}\right)$ потенциал был представлен в следующем виде:

$$
U_{I R}(R)=\sum_{k=0}^{n} a_{k} x^{k}
$$

где $x=\frac{R-R_{m}}{R+b R_{m}}, a_{i}$ - подгоночные коэффициенты, $R_{m}-$ расстояние, близкое к равновесному. Явный вид параметров в выражениях (1)-(4) дан в [10].

Используя выражения (1)-(4) и входящие в них величины, приведенные в [10], в данной работе построены потенциалы взаимодействия интересующего нас димера. На рис. 1 приведены зависимости синглетного и триплетного термов KLi в зависимости от межьядерного расстояния в атомной системе единиц. Таким образом, зная синглетный и триплетный термы димера KLi в основном состоянии, можно перейти к расчету комплексных сечений спинового обмена.

\section{Расчет комплексных сечений спинового обмена при столкновении атомов калия и лития}

При столкновении двух щелочных атомов образуется квазимолекула, которая может быть описана соответствующими потенциалами взаимодействия. При столкновении атомных частиц, обладающих ненулевым электронным спином (в данном случае электронный спин $S=1 / 2$ ), происходит обмен электронами. При этом если один из сталкивающихся атомов обладал до столкновения электронной поляризацией (полученной, например, при оптической ориентации), то возможна также передача этой поляризации партнеру столкновения. Это хорошо известный процесс спинового обмена [7], его можно представить в следующем виде:

$$
A(\uparrow)+B(\downarrow) \rightarrow(A B) \rightarrow A(\downarrow)+B(\uparrow) .
$$

Стрелки условно указывают направление углового момента атома (в нашем случае - электронного спина).

Процесс спинового обмена можно описать в рамках формализма комплексного сечения [6]:

$$
q^{A B}=q^{-A B}+i q^{=A B} .
$$

Комплексное сечение спинового обмена можно представить в следующем виде через матрицы рассеяния $T$ :

$$
q^{A B}=\frac{\pi}{k_{A B}^{2}} \sum_{l=0}^{\infty}(2 l+1)\left[1-T_{0}^{A B}(l) T_{1}^{A B}(l)^{*}\right]
$$

Здесь $k_{A B}^{2}=\mu_{A B} v_{A B} / h-$ волновой вектор, $\mu_{A B}-$ приведенная масса сталкивающихся частиц $A$ и $B, v_{A B}-$ средняя относительная тепловая скорость сталкивающихся атомов, звездочка означает комплексное сопряжение. Матрица рассеяния в свою очередь может быть представлена через фазы рассеяния $\left(\delta_{S}^{A B}(l)\right)$ в канале с полным спином $S$ следующим образом:

$$
T_{S}^{A B}(l)=\exp \left(2 i \delta_{S}^{A B}(l)\right),
$$

где $l$ - орбитальное квантовое число.

Принимая во внимание (7) и (8), действительную и мнимую части комплексного сечения можно представить в виде

$$
\begin{gathered}
q^{-A B}=\frac{\pi}{k_{A B}^{2}} \sum_{l=0}^{\infty}(2 l+1) \sin ^{2}\left[\delta_{1}^{A B}(l)-\delta_{0}^{A B}(l)\right], \\
q^{=A B}=\frac{\pi}{k_{A B}^{2}} \sum_{l=0}^{\infty}(2 l+1) \sin 2\left[\delta_{1}^{A B}(l)-\delta_{0}^{A B}(l)\right] .
\end{gathered}
$$

Отсюда, зная синглетный и триплетный потенциалы взаимодействия димера $\mathrm{LiK}$, можно рассчитать фазы рассеяния на синглетном и триплетном термах и определить интересующие нас сечения.

Фазы рассеяния рассчитывались в квазиклассическом приближении в интервале энергий столкновения $E=10^{-4}-10^{-2}$ a.u. с использованием приближения Джефриса, модифицированного Ламбером [11]:

$$
\delta_{s}=\int_{R_{0}^{\prime}}^{\infty} F_{1}(R) d R-\int_{R_{0}^{\odot}}^{\infty} F_{0}(R) d R,
$$

где

$$
\begin{gathered}
F_{1}^{S}(R)=\left[2 \mu\left(E-V_{S}(R)-\frac{(l+1 / 2)^{2}}{2 \mu R^{2}}\right)\right], \\
S=s, t, q, \\
F_{0}(R)=\left[2 \mu E-\frac{(l+1 / 2)^{2}}{R^{2}}\right] .
\end{gathered}
$$




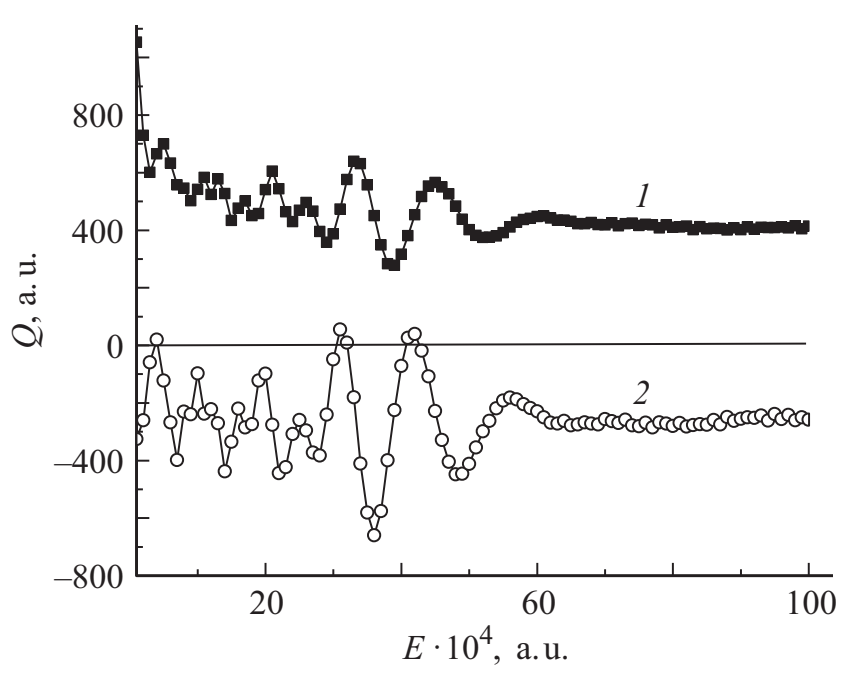

Рис. 2. Зависимость действительной $(1)$ и мнимой (2) частей комплексного сечения спинового обмена от энергии столкновения в системе двух атомов ${ }^{39} \mathrm{~K}-{ }^{7} \mathrm{Li}$ (атомная система единиц).

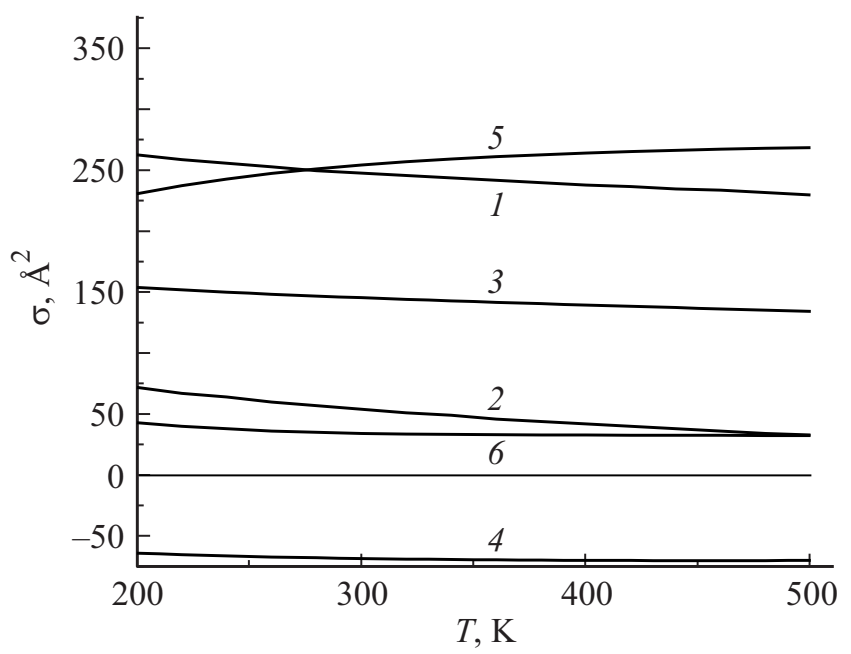

Рис. 3. Зависимость от температуры действительной $(1,3,5)$ и мнимой $(2,4,6)$ частей сечения спинового обмена при столкновении атомов ${ }^{39} \mathrm{~K}$ со щелочными атомами: система $\mathrm{K}-\mathrm{K}[12](1,2)$, система $\mathrm{K}-\mathrm{Li}(3,4)$, система $\mathrm{K}-\mathrm{Na}[6](5,6)$.

Здесь $E-$ кинетическая энергия, $R_{0}$ и $R_{0}^{\prime}-$ корни уравнений $F_{1}^{S}(R)=0, F_{0}(R)=0$ (для $F_{1}^{S}$ берется больший корень), $V_{S}(R)$ - потенциал взаимодействия, соответствующий полному спину $S$.

На рис. 2 представлены полученные в результате расчета зависимости действительной $q^{-}$и мнимой $q^{=}$ частей комплексного сечения от энергии столкновения. Как видно из рис. 2, сечение сдвига частоты KLi находится в области отрицательных значений.

На рис. 3 приведены усредненные по скоростям зависимости действительной и мнимой частей сечения спинового обмена в интервале температур $T=200-500 \mathrm{~K}$. Кроме данных по системе KLi на рисунке для сравнения приведены результаты расчета сечений столкновений атомов $\mathrm{K}$ с другими щелочными атомами (K [12] и $\mathrm{Na}[6])$. Как видно из рисунка, сечение сдвига для системы $\mathrm{K}$-Li имеет отрицательное значение во всем интервале рассматриваемых температур.

Таким образом, полученные в настоящей работе температурные зависимости действительной и мнимой частей сечения спинового обмена позволяют рассчитать вклад спин-обменного процесса при столкновении между поляризованными атомами калия в ширину линии магнитного резонанса и в сдвиг линии магнитного резонанса.

\section{Конфликт интересов}

Авторы заявляют, что у них нет конфликта интересов.

\section{Список литературы}

[1] Величанский В.Л., Козлов А.Н., Живун Е.В. и др. // Успехи современной радиоэлектроники. Зарубежная радиоэлектроника. 2013. Т. 12. С. 43-59.

[2] Александров Е.Б., Вершовский А.К., Пазгалев А.С. // ЖТФ. 2006. Т. 76. № 7. C. 103; Aleksandrov E.B., Vershovskii A.K., Pazgalev A.S. // Tech.Phys. 2006. V. 51. N 7. P. 919.

[3] Александров Е.Б., Балабас М.В., Вершовский А.К., Пазгалев А.С. // ЖТФ. 2000. Т. 70. № 7. С. 118; Aleksandrov E.B., Balabas M.V., Vershovskii A.K., Pazgalev A.S. // Tech.Phys. 2000. V. 45. N 7. P. 931.

[4] Александров Е.Б., Вершовский А.К // УФН. 2009. Т. 179. № 6. C. 605-637; Aleksandrov E.B., Vershovskii A.K. // Phys.Usp. 2009. V. 52. N 6. P. 573.

[5] Alexandrov E.B., Balabas M.V., Pazgalev A.S., Vershovskii A.K., Yakobson N.N. // Laser Physics. 1996. V. 6. N 2. P. 244.

[6] Картошкин В.A. // Опт. и спектр. 2010. Т. 109. № 5. С. 733; Kartoshkin V.A. // Opt. Spectrosc. 2010. V. 109. N 5. P. 674.

[7] Happer W., Jau Y.-Y., Walker T. Optically Pumped Atoms. WILEY-VCH , 2010. 246 p.

[8] Дмитриев С.П., Доватор Н.А., Картошкин В.А., Окуневич А.И. // Опт. и спектр. 1994. Т. 77. № 5. C. 712-713; Dmitriev S.P., Dovator N.A., Kartoshkin V.A., Okunevich A.I. // Opt. Spectr. 1994. V. 77. N 5. P. 712.

[9] Картошкин В.A. //Опт. и спектр. 2010. Т. 108. № 6. C. 914-917; Kartoshkin V.A. // Opt. Spectrosc. 2010. V. 108. N 6. P. 866.

[10] Tiemann E., Knöckel H., Kowalczyk P., Jastrzebski W. et al. // Phys. Rev. A. 2009. V. 79. P. 042716.

[11] Мотm Н., Месси Г. Теория атомных столкновений. М.: Мир, 1969. 756 с.

[12] Картошкин В.A. // Опт. и спектр. 2011. Т. 111. № 6. C. 924-927; Kartoshkin V.A. // Opt. Spectr. 2011. V. 111. N 6. P. 881. 